\title{
Borde costero resiliente: aprendizaje post-27F para el norte grande de Chile
}

\author{
Resilient Coastal Rim: Post-27F Learning for the Big North of Chile
}

Hans P. Intveen Pérez, ${ }^{1}$ Arq.

${ }^{1}$ Ministerio de Vivienda y Urbanismo, Región de Antofagasta. Arquitecto y urbanista,coordinador de diseño en proyectos de restauración urbana en el proceso de reconstrucción post-27F, regiones del Maule y Bio - Bio, Chile. Correspondencia:hintveen@gmail.com/hintveen@minvu.cl

Recibido: 15 de agosto de 2016. Aceptado: 15 de octubre de 2016

Intveen, H. (2016) Borde costero resiliente: aprendizaje post-27F para el norte grande de Chile. Procesos urbanos Número 3, Ene-Dic. 24-37. Doi:10.21892/2422085X.265

\section{RESUMEN}

Con posterioridad al terremoto y tsunami de 2010 en Chile, se ha evidenciado que los espacios residuales que el crecimiento urbano no planificado de las ciudades ha dejado en el paisaje costero, se han transformado en la primera línea de defensa contra los eventos naturales catastróficos, al tener una relación directa con las unidades de paisaje donde se produce la mayor destrucción. El proceso de reconstrucción, posterior al desastre, ha permitido incorporar nuevos conceptos a la planificación del paisaje costero, lo que ha generado una experiencia y un caudal de conocimiento que permiten extraer principios de diseño urbano que pueden replicarse en otras geografías vulnerables, como el caso del Norte Grande en Chile. La investigación plantea una sistematización de principios generales de ordenamiento espacial para espacios costeros en el contexto de paisaje nortino, de una manera que permita no solo la resistencia al evento, sino su eficiente reconstrucción y rápida adaptación a circunstancias adversas.

Palabras clave: Mitigación, resiliencia, borde costero, paisaje urbano.

\section{ABSTRACT}

Following the earthquake and tsunami of 2010 in Chile, it has become evident that the residual spaces that unplanned urban growth of the cities has left in the coastal landscape have become the first line of defense against catastrophic natural events, since they have a direct relationship with the landscape units where the greatest destruction occurs. The post-disaster reconstruction process has allowed the incorporation of new concepts into coastal landscape planning, which has generated an experience and a wealth of knowledge that allow the extraction of principles of urban design that can be replicated in other vulnerable geographies, such as the case of the Great North in Chile. The research proposes a systematization of general spatial planning principles for coastal spaces in the context of the northern landscape, in a way that allows not only the resistance to the event, but also its efficient reconstruction and rapid adaptation to adverse circumstances.

Keywords: Mitigation, resilience, coastal edge, urban landscape.

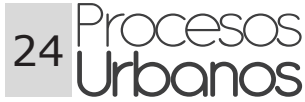


Intveen, H. - Borde costero resiliente: aprendizaje post-27F.

\section{INTRODUCCIÓN}

La relación entre las ciudades y el entorno natural siempre será un tema de interés, tanto en términos urbanos como antropológicos e incluso- poéticos, que se amplifica al momento de pensar en un entorno natural dinámico y cambiante como el paisaje costero, capaz de proveer espacio público de calidad y al mismo tiempo ser un ámbito de riesgo, sometido además a una presión tanto inmobiliaria como urbana creciente. En Chile, con posterioridad al terremoto y tsunami del 27 de febrero del 2010 (27F), ha sido necesario repensar esta relación, incorporando el conocimiento generado a raíz del intenso proceso de reconstrucción urbana que siguió a la catástrofe.

Resulta de especial interés entonces, analizar las lecciones de carácter estructural, y que son por ende traspasables a contextos que en este momento se encuentran en una situación de mayor vulnerabilidad que la zona ya afectada. El presente estudio se enmarca precisamente en la necesidad de traspasar el conocimiento y práctica en términos de diseño urbano que se ha generado en las zonas afectadas por el tsunami del $27 \mathrm{~F}$, a otras geografías.

Uno de estos contextos prioritarios es la zona del norte grande de Chile, que además cuenta con amplias zonas de "silencio sísmico" (entre Tacna y la península de Mejillones, siendo más precisos), donde, por ejemplo, no se han aplicado criterios que vayan más allá de tener clara la línea de inundación. Es cierto que los avances en materia de prevención nos tienen a la vanguardia en términos -incluso- mundiales, pero se trata de un aprendizaje cultural que no se ha traspasado enteramente al ámbito físico de la ciudad.

Así, cuando se examinan los Planes Reguladores Comunales (PRC) de ciudades costeras de la zona norte, se ve el riesgo como una situación peligrosamente simplista. Se establecen zonas de riesgo donde el peligro es absoluto, es decir, donde no se puede construir ni regularizar, lo cual no calza con la informalidad urbana de la realidad nortina, donde de todos modos se construye. Por otro lado, las zonas que no son de riesgo, para efectos de los planes, son $100 \%$ carentes de riesgo, es decir carecen de criterios relativos a la mitigación de estos. En algunos casos, las zonas de riesgo están a un metro de distancia de otra sin riesgo, de tal forma que se da la situación anómala de que una vivienda sin riesgo puede ser vecina de una riesgosa $e$, incluso más, que una misma vivienda está sobre un lote mitad riesgoso y mitad seguro. Esta falta de gradualidad o de una visión sistémica del problema es inherente a los instrumentos de planificación territorial.

El 27F dejó una serie de planes para las localidades afectadas que -al menosestablecían un complemento a la regulación costera y generaron áreas nuevas de interés para planificaciones futuras. Este nuevo esquema se ha aprovechado para alojar una serie de proyectos que han potenciado el espacio público de estos bordes costeros y fluviales. Pese a que su intención original $-y$ el fundamento para su alta inversión- está relacionado con la mitigación, su mayor aporte ha sido generar una nueva relación con el paisaje, que de alguna forma todavía está en proceso, ya que por la envergadura de estas iniciativas, se trata de proyectos que se consolidarán a lo largo de años, no necesariamente en la misma forma en que fueron concebidos.

La hipótesis preliminar que mueve a esta investigación es que las soluciones tipo que se generaron para estos territorios (barreras vegetales, por ejemplo) no son igualmente aplicables en todos los contextos, pero muchos de los conceptos que las sustentaron son válidos para otros paisajes, particularmente en bordes costeros y fluviales, y pueden ser un aporte para la relación entre ciudad y entorno natural.

En otras palabras, así como hay conceptos espaciales que son propios de ciertas geografías, hay reglas que son transversales a todas las situaciones, y este estudio es un paso en esa dirección.

\section{Las barreras naturales como solución resiliente para el borde costero}

La aproximación que se tuvo en el caso del proceso de reconstrucción en Chile en orden a crear una "barrera natural" o zona de mitigación, nace de la aproximación a la

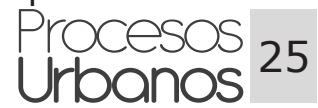




\section{Procesos Urbanos N³ Enero - Diciembre; 2016}

experiencia internacional adaptada a la singular realidad local. Hay que tener en cuenta que en el caso de Chile, se trata de un país que posee una intensa -y extensa- actividad volcánica con 2000 volcanes, 500 de ellos activos, cuya área de influencia abarca al menos el $30 \%$ de la geografía nacional. Adicionalmente, se trata de uno de los países con mayor actividad sísmica en el mundo, con un promedio de 200 temblores diarios y el registro de al menos un terremoto superior a $8^{\circ}$ Richter en los últimos cinco siglos, incluyendo el más grande de la historia documentada (1960, Valdivia). Como reconoce la jefa de la División de Desarrollo Urbano (DDU) del Ministerio de Vivienda, "Ante este difícil panorama, la alternativa que usualmente se plantea de 'no construir en zonas de riesgo' pierde todo sustento". (Giménez, en MINVU, 2013, p.14).

La experiencia de Japón, que es uno de los países que más ha avanzado en relación a la protección del borde costero, ha privilegiado los bosques monofuncionales (sin otros usos), basados en una tradición en el desarrollo de barreras vegetales que tiene características ancestrales, remontándose más de cuatro siglos. Su establecimiento se orientó originalmente a contener tormentas de arena, vientos salinos, marejadas y tsunamis, usando preferentemente pino negro japonés. En ciertas zonas de Japón, la gente se ha acostumbrado a este tipo de paisaje, llamamándo "hakusaseisyou", que significa "hermosa costa con pinos y playas arenosas" (Shaw et. al., 2012, p. 3).

Uno de los casos paradigmáticos se encuentra en la bahía de Sendai, donde un distinguido señor feudal llamado Masamune Date, comenzó a plantar pino negro japonés alrededor del canal Teizan en la planicie de Sendai, alrededor del siglo XVII. Se trataba de proteger a la gente que vivía en zonas dunarias costeras y que habían sufrido los rigores de las tormentas de arena y cambios en las mareas. Para efectos de generar una gestión eficiente, Masamune permitió que la gente pudiese vender leña de las ramas que hubiesen caído o estuviesen quebradas y de esta forma cubrir los costos de mantenimiento. De esta forma, se consolidaron bosques que se han mantenido vigentes por más de cuatro siglos, lo que ha generado zonas de protección que varían de los 200 a 400 metros.

No se trata de un caso aislado, en la ciudad costera deTohokusucedióalgosimilar(Imamura \& Anawat, 2012, p. 21 -30), y las comunidades locales mantuvieron esta infraestructura verde por más de 350 años mediante eventos como festivales que conmemoraban la iniciativa original. En ambos casos, el respaldo y adopción de la idea por parte de la ciudadanía resultaron fundamentales. Así, estas iniciativas comunitarias fueron progresivamente generando un interés estatal por consolidar y formalizar la existencia de estas áreas, lo que llevó a que ya entrados en el siglo XX se generara un programa de forestación que se intensificó aún más a mediados de siglo, luego del terremoto y tsunami de Chile en 1960, cuyo oleaje también afectó a Japón.

En la actualidad, el tsunami del 11 de marzo demostró que, pese a sufrir severos daños, esta infraestructura verde ayudó a reducir el impacto del oleaje (Shaw et. al. 2012, p. 5). Asimismo, demostró -en el sentido contrarioque sobre cierta envergadura, no existe medida de mitigación que sea $100 \%$ segura.

Se han documentado casos esperanzadores en tsunamis bajo los 9 metros en los cuales la barrera vegetal logró retardar el impacto del tsunami y protegió numerosos hogares mediante la captura de escombros y trozos de barcos. Por ejemplo, en la ciudad de Hachinoe en la prefectura de Aomori, el bosque logró capturar al menos 20 barcos que fueron transportados por una ola que alcanzó los 6 metros y evitó que se estrellaran contra las viviendas. Las viviendas, eso sí, fueron inundadas por 3 metros de agua, pero mantuvieron su integridad como tales.

Resumiendo la experiencia de Japón, la instauración de estas barreras se ha validado como política en el borde costero, pero han entendido asimismo que es necesario complementarlas con obras civiles que mejoren su eficiencia y permitan su continuidad en el tiempo. En ese mismo sentido, el gobierno de la prefectura de Miyagi recomienda al menos 3 criterios. Primero, coordinarse con otras acciones en el territorio, como diques y obras de manejo de escombros. Segundo, seleccionar 
Intveen, H. - Borde costero resiliente: aprendizaje post-27F.

\section{Emplazamiento de las zonas de estudio}

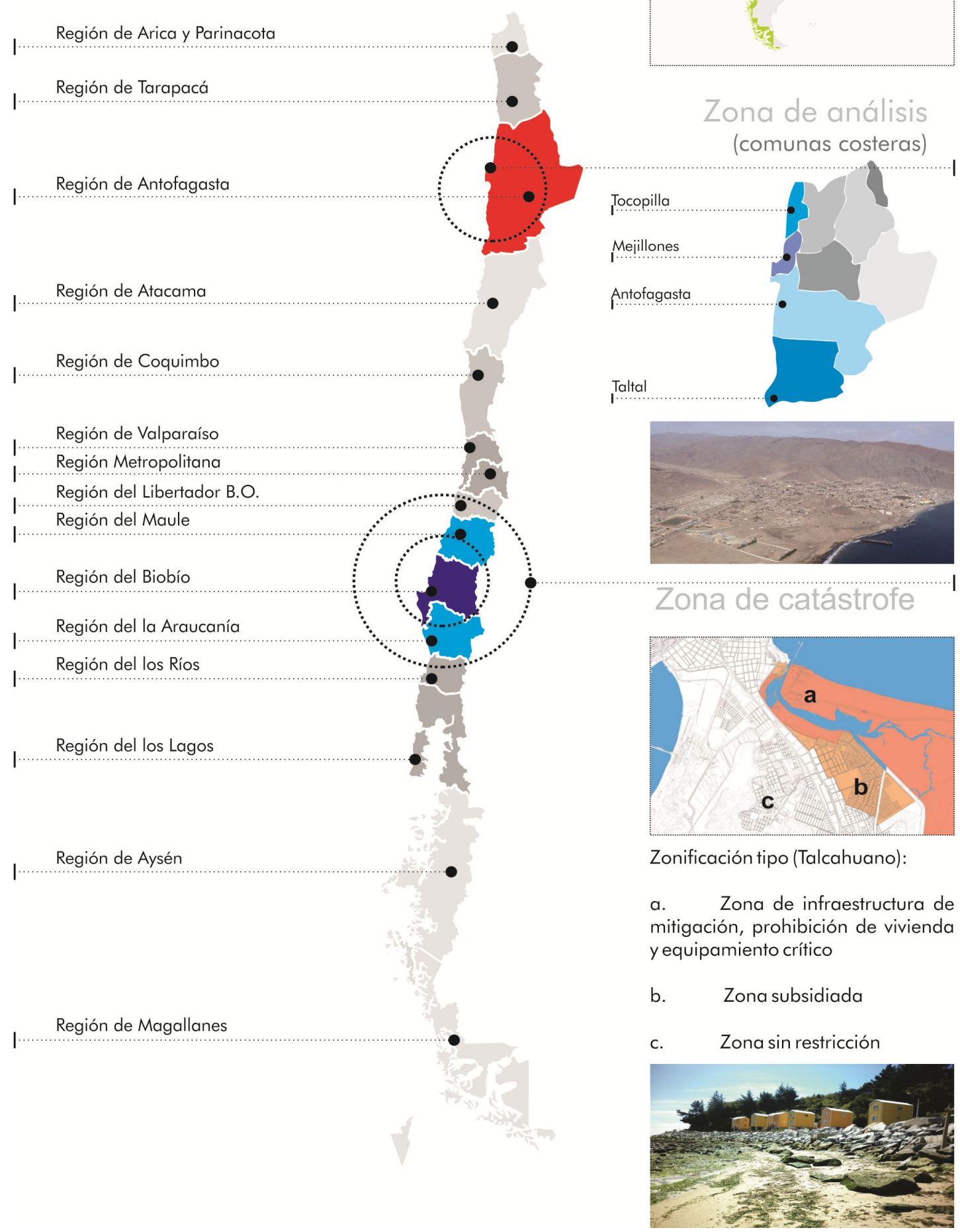

Figura 1. Emplazamiento de las zonas principales afectadas por la catástrofe (Maule, Biobío) y la zona desértica a analizar (Antofagasta) Fuente: elaboración propia. 
y complementar con especies que se adapten a las condiciones locales y que colaboren en la biodiversidad. Tercero, colaborar con ONG, voluntarios y sector privado para apoyar la sostenibilidad en términos de mantenimiento.

Como se puede apreciar, en el caso japonés, desde sus inicios, estas barreras vegetales fueron entendidas como una "infraestructura" antes que un espacio público.

A comienzos del proceso de reconstrucción en Chile, uno de los criterios principales que se planteó para el borde costero fue aplicar la resistencia naturalquese había logrado observar en algunas experiencias exitosas de la zona asiática. Eso sí, con algunas modificaciones, ya que una de las características más llamativas del proceso chileno de reconstrucción guarda relación precisamente que - a diferencia de la tendencia japonesa- con el hecho de que se realizó a través del espacio público, que en este caso se planteó como objetivo adicional la resiliencia.

Este esfuerzo de unir conceptos de resiliencia (mitigación) y criterios de espacio público se refleja con claridad en una reciente entrevista a Alejandro Aravena (Elemental), autor del Plan Maestro para Constitución (una de las ciudades más afectadas por el tsunami) y el más reciente ganador del premio PRITZKER.

La solución fue crear un bosque entre la ciudad y el mar, capaz de resistir y disipar la fuerza de la naturaleza. No sólo reducía la energía de las olas, sino que además evitaba la inundación de la ciudad y mejoraba la proporción de espacio público de 2 a 7 metros cuadrados por habitante.(Seitz, 2016).

Así, en la nueva planificación del espacio costero, se generó una estrategia de "cinturones vegetales" que servirían como un auténtico "frente de ataque" ante los tsunamis y marejadas, según los criterios emanados de numerosos informes especializados (Tanaka, 2010) que hacen referencia a la "función protectora de los manglares y otros bosques costeros sobre vidas humanas y los bienes", referidos a la zona de Sumatra (Rodriguez y Gajardo, 2011) luego del gran tsunami de Indonesia, el 2004.
Con base en esta evidencia empírica, el Estado chileno abordó el proceso de reconstrucción con un "Plan de Acción" (MINVU, 2013, p.32) que en el ámbito urbano tuvo tres objetivos principales para la zona de catástrofe: Primero, definir una zonificación adecuada de las piezas urbanas costeras; diferenciando áreas de riesgo con usos restringidos, áreas con usos condicionados y áreas excluidas de riesgo. Segundo, Asegurar vías de evacuación y establecer zonas seguras. Tercero, planificar la localización del equipamiento crítico.

Como complemento a esta zonificación, se planificaron "barreras vegetales" en el borde costero, aunque con algunas limitaciones que los mismos estudios fueron configurando. Por ejemplo, estas barreras deben tener un ancho no menor a $20 \mathrm{~m}$ de masa vegetal con una distancia de 1,6 metros entre árboles (1600 o más especies por hectárea), y un diámetro de tronco mayor a $13 \mathrm{~cm}$ (Tanaka, 2010). Adicionalmente, estos mismos estudios admiten que con profundidades de inundación superiores a los $5 \mathrm{~m}$., la efectividad de estos cinturones verdes decrece $y$, en el caso de que los cinturones sean demasiado angostos (menos de $3 \mathrm{~m}$, es decir, dos corridas de árboles) incluso se transforman en un riesgo adicional debido a que el mismo desprendimiento de árboles genera una destrucción adicional. En el caso de India, por ejemplo, estos conceptos han generado toda una política de generación de "bioshields" (Feagin et. Al., 2009, p. 1-11) o escudos naturales que, de manera sorprendente, tienen como protagonistas algunas de las mismas especies que se ocuparon en Chile; la Casuarina equisetifolia y la Acacia para zonas de bosque y la Ammophila para estabilización dunaria.

Cómo se puede apreciar, tanto la experiencia en Asia como en Chile ha generado todo un fructífero campo de investigación, pero su aplicación sigue estando restringida a zonas que han sufrido catástrofes, antes que a zonas que las sufrirán en el futuro cercano. De hecho, en el mismo caso chileno, toda la zona norte (la más vulnerable) no ha aplicado de forma total estos criterios, salvo lo que se refiere a la logística de alerta y evacuación que, pese a ser quizás el criterio más relevante, debe estar acompañada -idealmente- por un entorno que haga más eficiente el accionar y que permita, además, recuperarse con mayor rapidez. 
En ese sentido, la pregunta sigue siendo la misma ¿Cómo llevar todo este aprendizaje a un contexto distinto? En los siguientes capítulos se intentará responder esta pregunta para el norte grande, empleando 3 ámbitos de conocimiento: Lo abstracto, lo físico y lo existencial, de acuerdo con la categorización que hace Munizaga (1997) con respecto al espacio público urbano (Tabla 1 ).

\section{Resultados y aprendizajes}

Hablar de un "bosque urbano" parece ser un auténtico contrasentido. De hecho, en el proceso de reconstrucción del borde costero post 27F, la mayor parte de los parques que fueron concebidos originalmente como "bosques de mitigación" terminaron siendo auténticos parques, y es difícil que se logren las densidades inicialmente diseñadas (1600 especies por hectárea) para estos paños, pese a la voluntad de las autoridades y diseñadores. Esto es aún más crítico en los casos para los cuales se proyectaron bosques cerrados (incluso enrejados) aledaños a sectores habitados.

La solución, que de alguna forma el tiempo se encargará de consolidar, se acerca más al concepto de un bosque de densidad media inserto en un parque, y que el este, con su estructura de recorridos genere la conexión urbana necesaria para que sienta parte del entorno habitado, ya que, incluso cuando se trate de un bosque armónicamente inserto en un parque, debe ser una masa verde que permita su uso y ser asumido por la localidad como un espacio útil y no como un área muerta.

Ahora, todo este esfuerzo de diseño -y política pública- no pasaría de ser una singularidad si el conocimiento no es aplicable a otras latitudes, de tal forma que aparece como válida y sustancial la pregunta que origina esta línea de estudio: ¿Qué sucede si cambiamos el paisaje? Más aun, ¿qué sucede si cambiamos radicalmente el paisaje, digamos... de una zona húmeda al desierto más árido imaginable?

Precisamente, en orden a obtener algunas respuestas que sean aplicables en todas las geografías, se ha tomado la situación espacial de ciudades costeras de la región de Antofagasta como Chañaral, Taltal, Antofagasta, Mejillones, Tocopilla e Iquique (y otros asentamientos de menor envergadura) que poseen enormes similitudes; todo esto en orden a comparar situaciones análogas de la región del Biobío, como Tirúa, Dichato, Cobquecura o Talcahuano.

El contrapunto es radical. Por ejemplo, en el caso del norte grande, la solución mediante bosque o barrera vegetal clásica no es practicable, por varias y poderosas razones, como mantenimiento, espacio, imposibilidad de lograr la densidad necesaria; pero es factible perfectamente, ampliar el alcance de lo que significa un bosque o una barrera vegetal, se puede dotar al concepto de una flexibilidad mayor, complementarlo. Así, si bien es cierto que el establecimiento de bosques con densidad como para mitigar tsunamis es impracticable, este es un impedimento solamente válido en lo que se refiere a la masa vegetal, no a la solución en términos abstractos, que es un área de protección con un componente natural o geográfico.

Entonces, pese a que en las ciudades costeras del norte grande existe un agudo problema de falta de espacio, al ser asentamientos relativamente estrangulados contra la cordillera de la costa, por su alta exposición y vulnerabilidad, estas ciudades nortinas

Tabla 1. Ámbitos de conocimiento.

\begin{tabular}{|c|c|c|}
\hline Lo abstracto & con relación a ideas abstractas & $\begin{array}{l}\text { Aspectos urbanos } \\
\text { Aspectos socio-económicos }\end{array}$ \\
\hline $\begin{array}{l}\text { Lo concreto } \\
\text { (físico espacial) }\end{array}$ & con relación a aspectos físicos y visuales & $\begin{array}{l}\text { Aspectos físicos } \\
\text { Aspectos bióticos } \\
\text { Aspectos paisajísticos }\end{array}$ \\
\hline Lo existencial & con relación al significado & $\begin{array}{l}\text { Aspectos socio-culturales } \\
\text { Participación ciudadana }\end{array}$ \\
\hline
\end{tabular}

Fuente: MUNIZAGA (1997). 


\section{Procesos Urbanos N³ Enero - Diciembre; 2016}

necesitan estrategias de protección con relación al borde costero. Es decir, este escudo natural sigue siendo necesario, pero debe pensarse de forma distinta.

Trasladando la lógica que se ocupó en los planes maestros de la zona sur con posterioridad al terremoto, una forma de protección mixta es transformar a la pieza urbana costera (lo vegetal más lo netamente urbano) en una barrera en sí misma, incorporando tanto espacio público como edificación resiliente, para generar lo que se denomina como un "bio-shield", un escudo mixto natural-urbano que funcione como una frontera urbanopaisajística, con un ordenamiento espacial que acentúe las características resilientes de la zona. Es precisamente este segmento y su ordenamiento espacial lo que interesa dilucidar.

¿Qué características deberían tener estas distintas líneas? ¿Cómo se debería ordenar espacialmente este bioshield? ¿Qué políticas o prácticas sociales deberían complementar este ordenamiento?

\section{Una visión abstracta-racional/re- configurar el paisaje}

Al momento de hacer un diagnóstico de la situación actual del borde costero del norte grande en términos de resiliencia, si se toman en cuenta los tres objetivos del "plan de acción" (MINVU, 2013) para la reconstrucción (zonificación, consolidación de vías de evacuación, localización de equipamiento crítico) se encuentran deficiencias importantes. En el caso del primer punto, ninguna de las localidades costeras cuenta con un plan maestro con este tipo de zonificación. En el caso del segundo, ya todas las comunas tienen vías de evacuación, pero tanto las vías como las zonas seguras no responden a un estándar particular (que además no se ha consensuado a nivel nacional). Por último, respecto al tercer punto, todavía existen muchos equipamientos críticos en zonas poco seguras, como establecimientos educacionales y departamentos municipales.

Ahora, al realizar el traslado de conocimiento a este nuevo paisaje, una de las configuraciones que permanece válida en relación con las barreras vegetales propuestas en la zona de catástrofe, es la estructuración con base en 30 Procesos líneas sucesivas de protección, que deben además conducir claramente hasta las zonas seguras. Desde un punto de vista abstracto (que se puede llevar a política urbana), esto se traduce en la estructuración -mediante una configuración resiliente- del territorio. Esto implica necesariamente una zonificación transversal del borde costero, que para efectos prácticos entenderemos como una zona de 500 metros desde la línea de alta marea y bajo los $10 \mathrm{~m}$. de altura, siguiendo de forma referencial como se indica en la tabla 2.

Tabla 2. Zonificación del territorio.

\begin{tabular}{cc}
\hline $\begin{array}{c}\text { Sobre línea de alta } \\
\text { marea }\end{array}$ & Zona \\
\hline $0-1 \mathrm{~m}$. & Riesgo crítico \\
$1-3 \mathrm{~m}$. & Riesgo alto \\
$3-5 \mathrm{~m}$. & Riesgo moderado \\
$5-10 \mathrm{~m}$. & Riesgo bajo \\
$10 \mathrm{~m}$. o más & Sin riesgo inminente \\
\hline
\end{tabular}

Fuente elaboración propia.

Para hacer más eficiente esta zonificación, se deberían incorporar incentivos tributarios para quienes apliquen construcción resiliente, en toda la zona bajo los 10 metros, focalizados a través de planes maestros para zonas con alta vulnerabilidad antrópica, y en los casos en que no exista información dura (como cartas de inundación) aplicar principios básicos de ordenamiento como los presentados en este documento. Bajo la misma lógica, resulta estrictamente necesario identificar instalaciones vulnerables que posean un peligro inminente en términos de contaminación, como plantas de energía, bodegas, combustible, etc., cuestión que en el caso del norte grande es una realidad crítica debido a la cantidad de pasivos ambientales que se ubican en el borde costero. En la zona de estudio, por ejemplo, Tocopilla y Mejillones poseen termoeléctricas, y el resto zonas industriales con residuos mineros.

Como también se logró apreciar en proceso de reconstrucción, es de la mayor importancia el poder generar equipos de trabajo municipales competentes para la aplicación y seguimiento de los planes maestros, que antes que ser un "dibujo" son herramientas focalizadoras de política pública. Para esto, es estratégico generar alianzas con universidades, que permitan orientar requerimientos constructivos especiales para las zonas vulnerables de 
acuerdo con la geografía e identidad local (Figura 2).

Sin perjuicio de zonificaciones más estrictas que puedan generar estudios especializados, los criterios relativos a las líneas sucesivas de mitigación que plantean los bosques, se pueden traducir en las siguientes secciones de protección, o situaciones espaciales que podrían componer este "escudo natural" o bioshield.

Inmediatamente aledaño al mar, es necesario configurar una $1^{\text {a }}$ línea de estabilización (cota 0 a 5) como primer frente de "ataque" hacia los requerimientos del paisaje. Dependiendo del contexto, puede tratarse de un sistema de estabilización de dunas mediante vegetación costera, como alternativa "blanda", o bien en los casos que el espacio no lo permita- de infraestructura "dura" como rompeolas, muros de contención costeros, etc. Con cualquiera de estas alternativas, el objetivo es la consolidación de una primera zona de rugosidad $y$, al mismo tiempo, la generación de una barrera salina y contención climática, así como mitigación de marejadas y contaminación producida por industrias (Figura 3).

Entrelazada con esta primera línea sigue una $2^{a}$ línea de protección (cota 0 a 10): planteada como una columna vertebral (una vía urbana costera de seguridad), que contemple rellenos y elementos que aporten rugosidad. Paños con especies arbóreas de mayor tamaño (que pueden crecer debido a la barrera anterior), complementada por elementos que puedan servir como disipadores y al mismo tiempo como mobiliario urbano resistente. Idealmente, debieran considerarse rellenos con una configuración fractal, que generen una segunda zona de rugosidad de mayor envergadura (Figura 3).

A continuación debería configurarse una $3^{a}$ línea de regularización (cota 5 a 20 aprox.), centrada en la re-estructuración del espacio público residual del borde costero. Incorporar la reutilización de estructuras abandonadas, tales como industrias y asentamientos precarios. Estos espacios públicos deben ayudar a generar puntos de conexión expeditos hacia las vías de evacuación. Como en este segmento ya se cuentan paños de vivienda, se pueden generar proyectos para la reutilización que aguas grises que permitan abastecer las áreas verdes que no queden al alcance del sistema de riego local (Figura 4).

La $4^{a}$ línea de consolidación (cota 10 a 30 aprox.) consiste en mobiliario y obras complementarias que consoliden las vías seguras, tanto de evacuación hacia las cotas de mayor altura como de descenso de material: doble solera, calles con bombeo inverso, etc. Debe incorporarse la generación de vías-cauce que permitan el desahogo de la pendiente, así como paños de drenaje integrados al paisajismo (Figura 5).

Por último, una $5^{a}$ línea de contención (cota 30). En esta zona se debiera generar una estructura de puntos de reunión, estratégicamente alejados de las quebradas, sobre vías estructurantes a medio cerro. Sobre esta estructura se generarían las canalizaciones de los escurrimientos, idealmente complementada por piscinas o micro-lagunas con valor urbano, o bien consolidar zonas de jardines secos que funcionen como puntos de drenaje. En esta zona también resulta relevante la protección de laderas mediante aterrazamientos (Figura 5).

\section{La visión física-espacial / re-escalar el paisaje}

Luego del ordenamiento espacial y legal de este segmento, desde un punto de vista netamente físico, este bioshield debe tener una medida, una escala precisa, transformándose además en un espacio público adecuado e informativo. De esta forma, al mismo tiempo de plantar bosques o consolidar manglares en la costa, es necesario educar acerca de la función que pueden cumplir ante adversidades.

Al igual que en la costa, es imperativo una red de espacios públicos de acogida en la cintura segura de la ciudad (en la 5 a línea mencionada antes), cosa que en el caso de las ciudades nortinas ( $y$ las ciudades chilenas en general) escasea, lo cual suele provocar situaciones de peligro extremo en los casos de emergencia, no por el evento sísmico en sí, sino por la confusión y descoordinación propia de una situación catastrófica. 


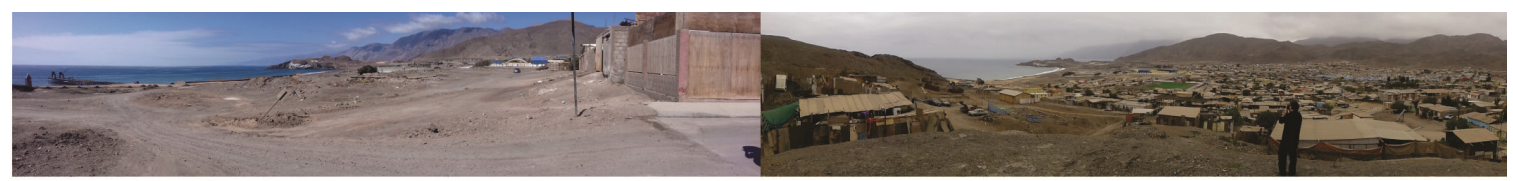

FOTOGRAFÍAS: a) Borde costero; b) muros de protección salitreros; c) Cierros irregulares y tomas de espacio público; d) intervenciones sin terminar; e) muros de contención sin consolidar; f) participación barrial en el diseño del espacio público.

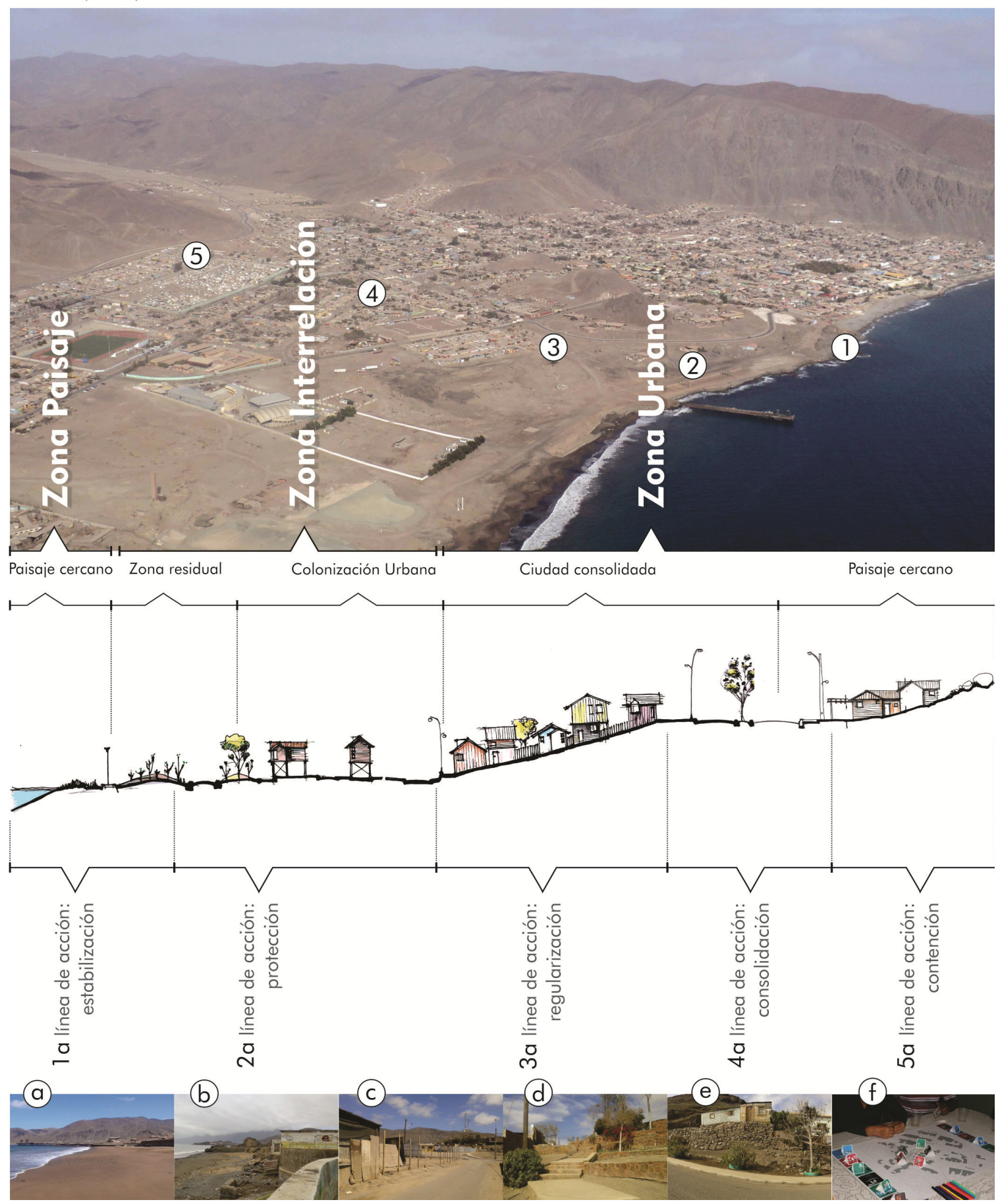

Figura 2. Arriba: Comparación de paisaje entre la zona de catástrofe de 2010 y la zona desértica analizada. Abajo: ordenamiento transversal de la pieza urbana costera. Fuente. Elaboración propia

\section{Urocesos}


Intveen, H. - Borde costero resiliente: aprendizaje post-27F.

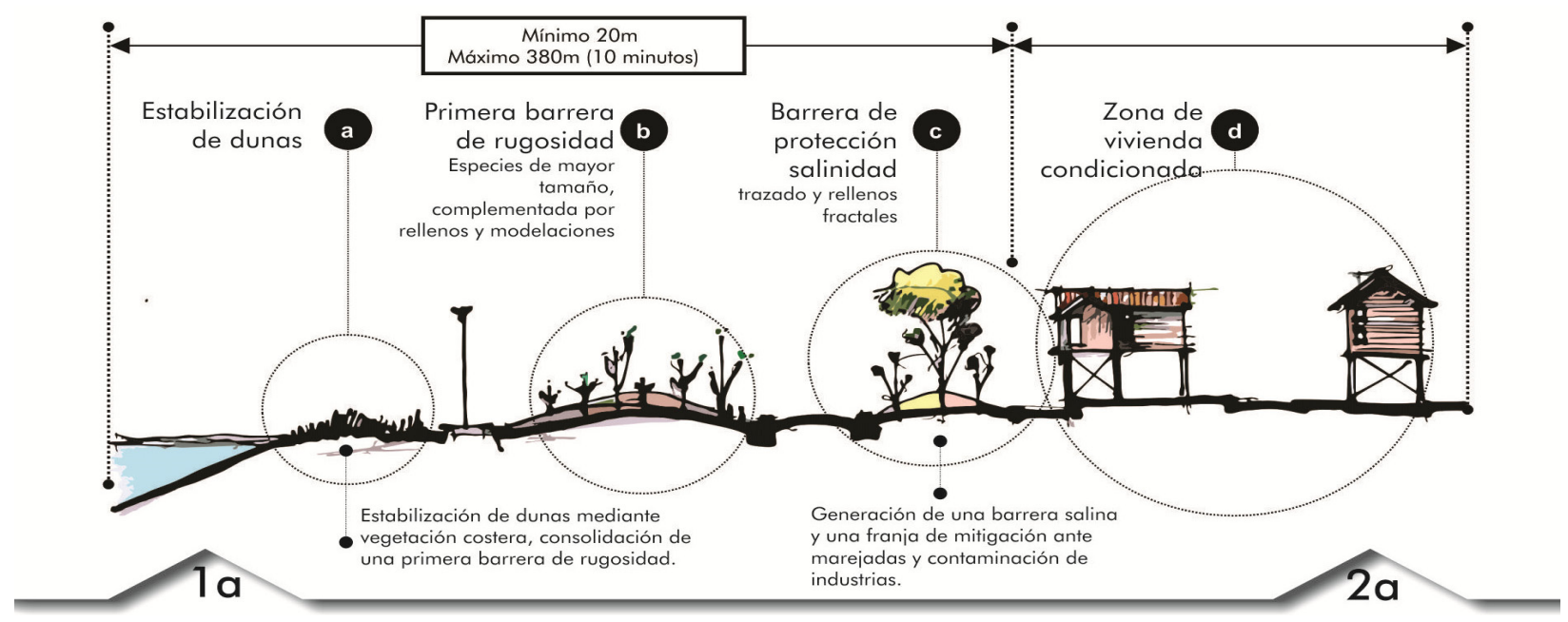

Figura 3. Esquema de la primera y segunda línea de la pieza urbana de mitigación.

Fuente: elaboración propia

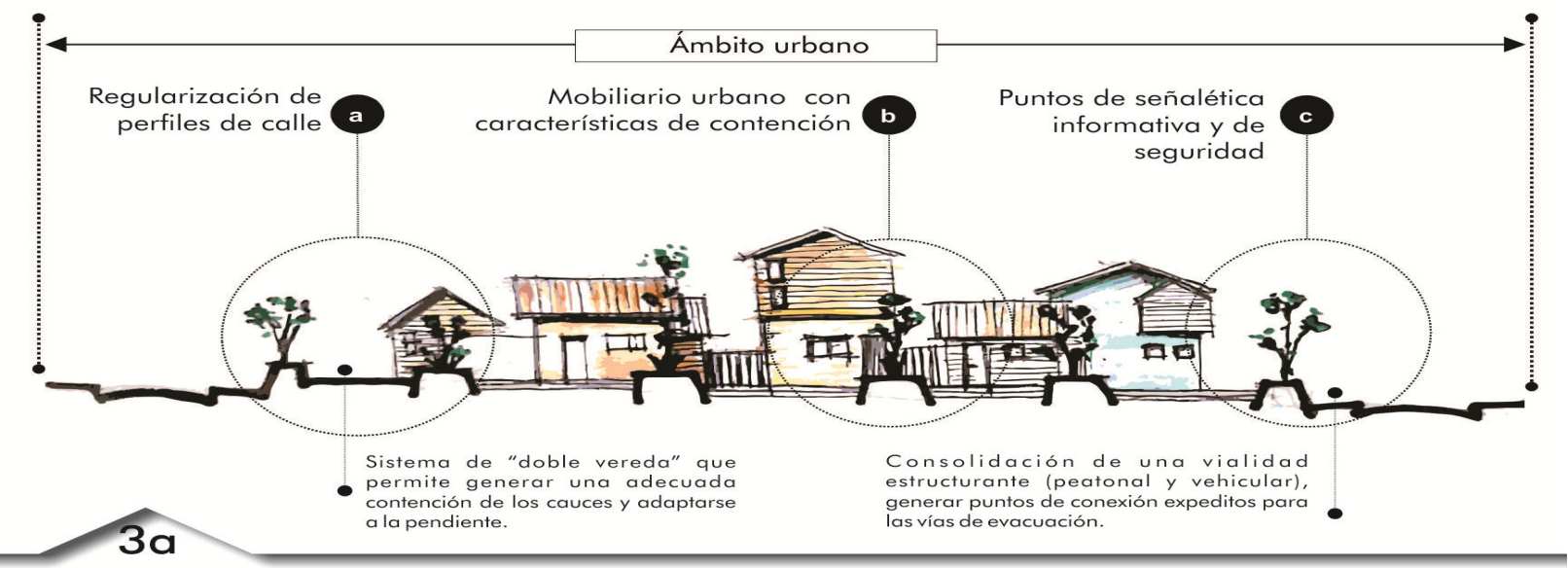

Figura 4. Esquema de la tercera línea de la pieza urbana de mitigación.

Fuente: elaboración propia

En los casos en los cuales, por razones de fuerza mayor, no existe la posibilidad de arrancar a una distancia segura en un lapso prudente, deberían, en su defecto existir alternativas verticales, los edificios cercanos deberían tener un plan de seguridad que incorpore la posibilidad de la evacuación vertical y la asistencia a personas ajenas al edificio (apertura eficiente de rejas y cerramientos).

Para la cuantificación de estos espacios en términos de distancias, es necesaria la incorporación de una variable extremadamente sensible: el tiempo. Si queremos traducir esta variable a espacio, resulta necesario tomar como referencia al ciudadano más desvalido, que vendría a ser una mujer llevando niños desplazándose a una velocidad aproximada de
2,52 km/hora (poco menos de un metro por segundo o 42 metros por minuto).

En el borde costero de la zona norte, la cota establecida para lograr un adecuado rango de seguridad se ha consensuado en los 30 metros, y se encuentra en promedio a unas 4 cuadras, es decir unos 500 metros aproximadamente. Esto significa que, en una emergencia, caminando a esta velocidad, una madre con su hijo se demoraría alrededor de 11 minutos en llegar a la cota de seguridad, lo cual se encuentra bajo el estándar de 15 minutos máximo que tenemos para evacuar un lugar cercano a donde se ha producido un sismo de gran magnitud. Pero esto es suponiendo que la persona en cuestión se encuentra directamente conectada con una vía de evacuación, lo cual 


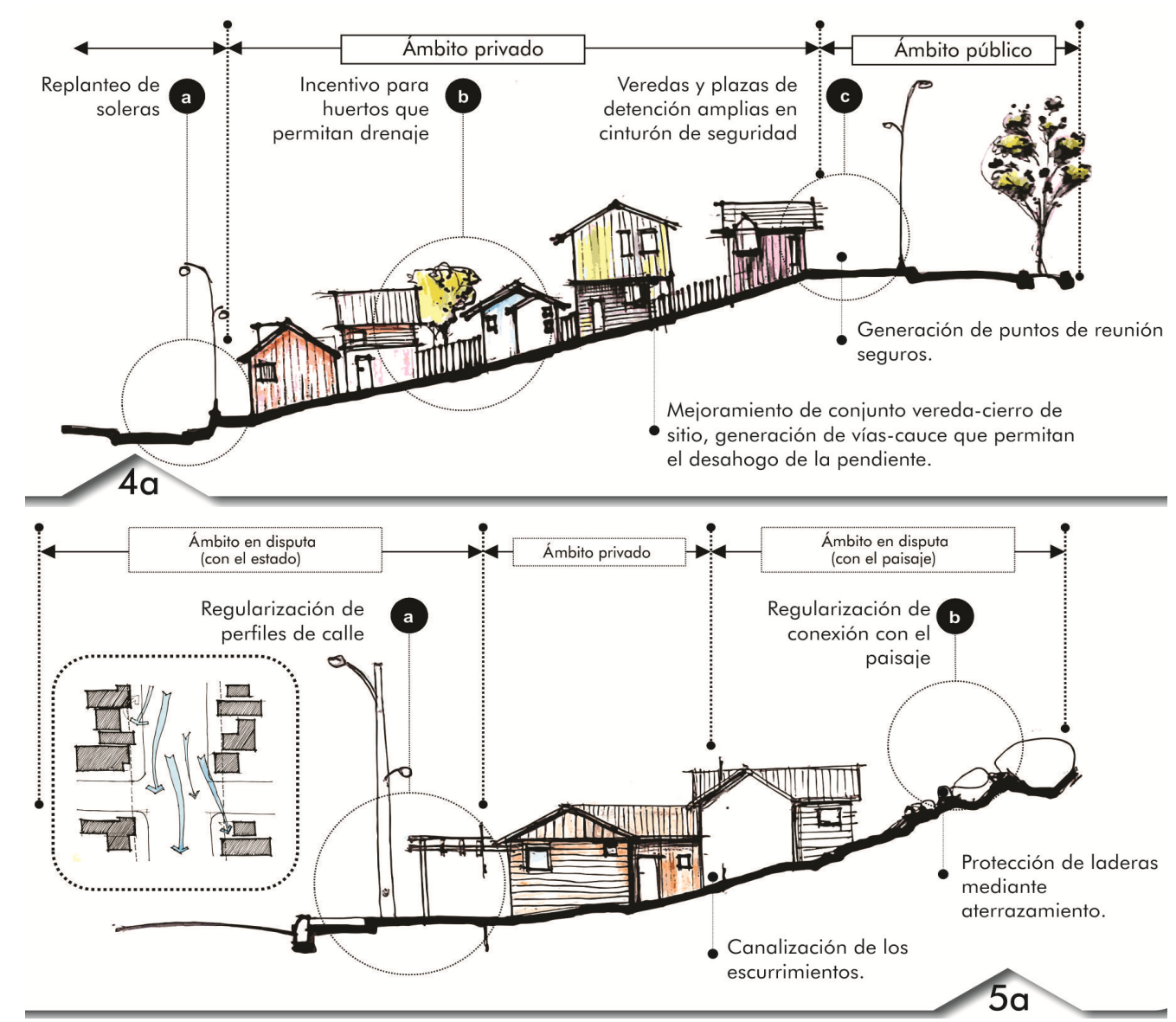

Figura 5. Esquema de la cuarta y quinta línea de la pieza urbana de mitigación.

Fuente: elaboración propia

no suele ser real en todo el desarrollo del borde costero. Usualmente hay que agregar un tiempo para llegar a esa vía, y según el estándar que hemos considerado, se trata del punto crítico a considerar: no puede haber una vía de evacuación a más de 4 minutos de distancia para un peatón.

Ahora, esto tiene consecuencias prácticas a nivel de espacio público, ya que se debería garantizar que la conexión con un punto de escape nunca sobrepase estos 4 minutos, lo cual se traduce en un rango que podría ir desde los 80 a 100 metros (con un máximo de 120) entre vías de desahogo del espacio de borde, planteando un ritmo en el sentido longitudinal del borde costero que no siempre es fácil de lograr ya que suelen ubicarse infraestructuras (a veces privadas) de gran envergadura que restringen las posibilidades de escape. Estas instalaciones deberían estar pensadas en su mismo diseño para ser flexibles y permitir que, en caso de evacuación, puedan ser permeables y permitan el paso de los habitantes. La flexibilidad funcional es una característica crítica para lograr un bioshield eficiente en ciudades cada vez más complejas y multifuncionales.

\section{La visión existencial / re-significar el paisaje}

Por último, el aspecto inmaterial de la prevención es quizás el de mayor relevancia, ya que, desde un punto de vista existencial, resignificar el borde costero como una zona crítica es una labor que sobrepasa lo técnico, como se ha entendido en Chile, involucrando a las organizaciones sociales y los actores locales. Aldunce y León traducen estas cualidades inmateriales en los siguientes conceptos: Gobernanza, empoderamiento y construcción de capacidades, participación comunitaria, coordinación institucional, planificación de contingencias (Aldunce y León, 2007, p. 36 -39). 
Pese a que existe la conciencia de potenciar estos aspectos, el interés en estos temas baja sensiblemente en los períodos de quietud, como afirma Lagos (2012, p. 8), "Ciertamente, en Chile resulta muy fácil olvidar". De esta forma, se necesita una política activa y periódica de reuniones de sensibilización para autoridades locales, "...ayudar a la gente a adquirir nuevas destrezas cívicas, con especial atención en aquellos con un bajo estatus en la comunidad" (Berke y Campanella, 2006, p. 205). Se debe configurar una red local a lo largo de la línea de costa con entrenamiento adecuado, tanto para agentes locales como para líderes comunitarios y situaciones especiales de vivienda colectiva, a través de campañas de educación pública que provean certificación. Debiera ser obligatorio para las juntas de vecinos y copropiedades el tener un plan de seguridad, por ejemplo.

Una de las formas de mantener el interés ciudadano, pese a las dificultades logísticas que implica, es la realización de simulacros periódicos, a distintas horas, con una dificultad incremental, en el cual participen las autoridades. Es decir, re-significar la ciudad implica repensar, lo cual forzosamente debe hacerse en conjunto, como una sola comunidad.

En el caso de las plazas de pequeña envergadura, es una situación común, por ejemplo, asignar a un vecino que se encargue del riego o alguna responsabilidad relativa a la plaza misma. En el caso de las zonas de seguridad, podría suceder algo similar, se puede asignar a una de las viviendas cercanas a los puntos de encuentro la responsabilidad de guardar equipamiento (frazadas, colchonetas, toldos, etc.) que permitan pasar algunas horas en ese lugar, en coordinación con la Junta de Vecinos del Sector. A todo nivel, uno de los mayores aprendizajes luego de la catástrofe del $27 \mathrm{~F}$ fue precisamente la relevancia de mantener el sentido de comunidad para estas situaciones, cuestión que, en ciudades de mayor envergadura, estaba por completo extraviado.

\section{Conclusiones}

Al revisar la aplicabilidad en el norte grande de Chile de los criterios empleados en la reconstrucción urbana pos $27 \mathrm{~F}$, se pueden relevar algunos aspectos que debieran ser estructurales en cualquier estrategia sobre el paisaje urbano, en los tres ámbitos del análisis que se emplearon.

Primeramente, en un sentido racionalabstracto, hay aspectos transversales a cualquier geografía y que parten desde la formulación misma de un proyecto urbano, como incorporar el concepto de equidad territorial, ya que las metodologías actuales tienden a privilegiar desmedidamente las zonas con alta densidad poblacional.

Luego, debe existir una línea conductora entre formulación, diseño e implementación de estos proyectos, para evitar que cada una de estas etapas sean compartimentos estancos, con protagonistas distintos. Para esto deben implementarse instancias formales permanentes de coordinación entre las distintas entidades y disciplinas que tienen tuición sobre el territorio, y que no solamente involucren al sector estatal, sino también al sector privado y la comunidad organizada. Esta integración es necesaria para lograr grados de flexibilidad en los espacios tanto públicos como privados del borde costero, en la perspectiva de lograr adaptabilidad para situaciones críticas (que permitan su uso o permiso de paso, por ejemplo). Ahora, justamente en relación con esta flexibilidad, resulta necesario cuidar la formalidad institucional de estos paisajes urbanos, resguardando una figura legal que ampare su creación e implementación (Plan maestro, seccional, etc.). Es mucho más eficiente incorporarlos a la planificación que luego tener que recurrir a normativas de excepción, especialmente si el objetivo es lograr un espacio costero que funcione como una secuencia gradual hacia el paisaje. Esta formalidad es funcional asimismo para generar continuidad política a través de instrumentos concretos. El establecimiento de bosques (o zonas) de protección es una labor que puede demorar décadas y debe sobrevivir múltiples administraciones.

En un sentido físico-espacial, una estrategia válida en cualquier paisaje ha sido el manejo de los tiempos de desplazamiento y sus consecuencias en el espacio, lo que ha generado una medida específica para estructurar los recorridos, dimensionar las áreas y plantear

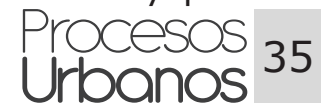


puntos de desahogo y conexión eficiente con las zonas seguras. En el caso específico de los tsunamis y marejadas, lo único que funciona $100 \%$ es la distancia, de tal forma que la primera prioridad es proveer de una red de espacios que permita lograr una distancia segura en caso de emergencia. En todo contexto es necesaria la complementación con el resto de las piezas urbanas, de tal forma que en conjunto presenten un grado de resistencia (ninguna pieza funciona por sí sola).

Siempre la vegetación debe jugar un lugar relevante, ya que los cinturones vegetales poseen un rol concreto, reducen la energía hidrodinámica, retrasan la llegada del oleaje y protegen las zonas habitadas a través de la captura de escombros y elementos contundentes. Además, las soluciones "blandas" o naturales deben privilegiarse debido a su sinergia, ya que los cinturones vegetales poseen beneficios complementarios para las comunidades costeras, como la protección contra tormentas, aire salino y tormentas de arena, y representan un espacio adecuado para la recreación y biodiversidad. Adicionalmente, ya se ha podido comprobar que las barreras vegetales pueden establecerse en casi cualquier entorno urbano, incluso en el desierto con base en una buena gestión de aguas grises.

En un sentido existencial, el involucramiento de la comunidad (o su simpatía por la iniciativa) son claves para el éxito y la perduración de las barreras vegetales y para la consolidación de un bioshield. Es perfectamente posible el involucramiento de las organizaciones sociales locales en la medida que obtengan tanto beneficios inmediatos como mediatos. El proceso de plantación puede ser abordado por el Estado, pero la supervivencia de los bosques debería ser una tarea social conjunta, no solo por un tema económico, sino por el sentido de pertenencia del espacio. Eso sí, para una adecuada adopción por parte de la ciudadanía, la barrera vegetal debe poseer otras funciones además de la meramente mitigadora, de tal forma que ostente legitimidad como espacio público, no solo como una mera infraestructura. En Japón, los campesinos han usado el bambú para generar ingresos adicionales mediante artesanías. Este valor social agregado es fundamental para que las comunidades locales puedan jugar un rol en el mantenimiento a largo plazo de los cinturones vegetales. En ese mismo sentido, es fundamental generar una red de apoyo en la comunidad con actores claves, tanto de los gobiernos locales como de las organizaciones de la sociedad civil. La labor en los colegios, por ejemplo, es fundamental; se trata de uno de los medios más eficientes para lograr una política de comunicación pública acerca de los beneficios de las barreras vegetales.

En definitiva, la participación de la comunidad debe configurarse como un involucramiento permanente, fomentado por autoridades y dirigentes sociales capacitados y comprometidos no solo con el espacio, sino con lo que este significa.

\section{Referencias}

Aldunce, P.; León, A. (2007). Opportunities for improving disaster management in Chile: a case study. [En línea]. Disaster Prevention and Management, Vol. 16 No. 1, 2007 pp. 33-41. Emerald Group Publishing Limited 0965-3562 DOI 10.1108/09653560710729794

Arenas, R.; Lagos, M; Hidalgo, R. (2010). Los Riesgos naturales en la planificación territorial. Centro de Políticas Públicas UC. Año 5, no.39, 2010. ISSN 0718-9745

Andrade, B.; Arenas, F.; Lagos, M. ( 2010). Incorporación de criterios de fragilidad ambiental y riesgo en la planificación territorial de la costa de Chile central. Rev. geogr. Norte Gd., Mayo 2010, no.45, p.520. ISSN 0718-3402

Berke, P.; Campanella, T. (2006). Planning forpostdisasterresiliency. TheANNALS of theamericanacademy of political and social science. March 2006 vol. 604 no. 1192-207. doi: 10.1177/0002716205285533 
Intveen, H. - Borde costero resiliente: aprendizaje post-27F.

Comisión Asesora Presidencial. (2013). Política Nacional de Desarrollo Urbano. Ciudades sustentables y calidad de vida. [En línea]. 6 de mayo, 2013. [Fecha de consulta: 01 diciembre 2013]. Disponibleen: http:// politicaurbana.minvu.cl/wp-content/uploads/2012/10/Documento Propuesta pol\%C3\%ADtica 2009. pdf\}

Feagin, R.A. ET AL. (2009). Shelter from the storm? Use and misuse of coastal vegetation bioshields for managing natural disasters. Department of Ecosystem Science \& Management, Texas A\&M University, College. 2009, p.1-11. doi: 10.1111/j.1755-263X.2009.00087.x

Lagos, M. (2012). Zonificación del riesgo de tsunami en el centro-sur de Chile. Rev. geogr. Norte Gd., Dic 2012, no.53, p.7-21. ISSN 0718-3402

Ikeuchi, K.; Isago, N. (2015) Earthquake disaster mitigation policy in Japan". Public Works Research Institute. [Fecha de consulta: 01 octubre 2015]. Disponible en: https://www.pwri.go.jp/eng/ujnr/ joint/39/paper/42ikeuchi.pdf

Imamura, F.; Anawat S. (2012). Damage due to the 2011 Tohoku earthquake tsunami and its lessons for future mitigation". Symposium on Engineering Lessons learned from the 2011 great east Japan earthquake, march 1-4, 2012, Tokyo, Japan. [Fecha de consulta: 01 octubre 2015]. Disponible en: http://www.jaee.gr.jp/event/seminar2012/eqsympo/pdf/papers/118.pdf

Intveen, H. (2008). Intervenciones en paisajes urbanos residuales: en búsqueda de una gramática para los espacios olvidados. Revista de Urbanismo, 17 (32), 102-126. doi:10.5354/0717-5051.2015.36172

Lagos, M.; Cisternas, M.; Mardones, M. (2008). Construcción de viviendas sociales en áreas de riesgo de tsunami. Revista de la Construcción, volumen 7 No2, pp. 4-16, 2008 [Fecha de consulta: 01 octubre 2015]. Disponible en: ftp://www.geo.puc.cl/geo/mlagoslo/Tsunami papers/Lagos et al tsunami housing 2008.pdf

MINVU. (2013). PLAN DE RECONSTRUCCIÓN Chile Unido Reconstruye Mejor. [En línea]. Santiago, 2010. [Fecha de consulta: 01 diciembre 2013]. Disponible en http://minvu.cl/opensite 20111122105648. aspx

MINVU. (2013). Reconstrucción urbana Post 27F: Instrumentos de planificación y gestión territorial. Ministerio de Vivienda y Urbanismo, División de Desarrollo Urbano, 2013. ISBN: 978-956-7674-81-7

Munizaga, G. (1997). Diseño urbano, teoría y método. Santiago de Chile, Ediciones UC, 1997. 336p. ISBN 9789561402935

NationaL Disaster Management Division. (2006). Protection and mitigation from risk of tsunami: A strategy paper. Ministry of Home Affairs Government of India, 2006. [Fecha de consulta: 01 octubre 2015]. Disponible en: http://nidm.gov.in/PDF/safety/flood/link1.pdf

Seitz, M.( 2016). Entrevista Alejandro Aravena. BBC Mundo, 2016. [Fecha de consulta: 01 septiembre 2016]. Disponible en: http://www.bbc.com/mundo/noticias/2016/04/160418_cultura_chile_ arquitectura_alejandro_aravena_premio_pritzker_ms

Shaw R.; Noguchi, Y.; Ishiwatari, M. (2012). Green Belts and Coastal Risk Management". World Bank Other Operational Studies, 2012. [Fecha de consulta: 01 octubre 2015]. Disponible en: http://wbi. worldbank.org/wbi/Data/wbi/wbicms/files/drupal-acquia/wbi/drm kn2-8.pdf 